\title{
TÉLÉGRAPHIE RAPIDE SYSTÈME POLLAK ET VIRAG ;
}

Par M. Désiré KORDA (1).

Deux ingénieurs hongrois ont trouvé que notre télégraphe actuel, cet appareil électrique qui symbolise pourtant, par excellence, la rapidité, n'est cependant pas assez rapide. Ils ont pensé que nos communications télégraphiques sont très chères, précisément parce qu'elles sont trop lentes.

Ce raisonnement a conduit à une invention remarquable M. Antoine Pollak, et son collaborateur, M. Joseph Virag, qui, malheureusement, n'a pas pu recueillir le fruit de son beau travail, ayant été enlevé par une mort prématurée le lendemain même de la besogne accomplie. Cette invention, que je dois vous présenter, est destinée à faire époque et à provoquer de profonds changements dans nos modes de transmission actuels.

Si je vous parle aujourd'hui de l'appareil Pollak-Virag comme d'une chose nouvelle, malgré les nombreuses descriptions qui en ont paru dans les périodiques et dans les comptes rendus des Congrès et même malgré que l'appareil, dans sa première forme, ait été exposé à l'Exposition Universelle de 1900, c'est que, depuis, il a subi de nombreux perfectionnements et est devenu d'une simplicité vraiment remarquable, comme vous pourrez l'apprécier vous-même, ét qu'enfin nous avons la bonne fortune d'avoir un de ses inventeurs parmi nous qui pourra faire fonctionner l'appareil devant vos yeux.

Le système combiné par MM. Pollak et Virag est un appareil de

(1) Communication faite à la Société française de Physique, séance du 7 décembre 1906. 
télégraphie rapide, écrivant directement, comme à la main, les lettres ordinaires de l'alphabet. Il diffère déjà en cela complètement de tous les autres appareils télégraphiques actuels. Il diffère en même temps par sa puissance incomparable permettant la transmission de 40.000 mots composés de plus de 250.000 lettres à l'heure, décuplant ainsi l'efficacité de l'appareil Baudot, qui jusqu'ici tenait la tête avec 5.000 mots à l'heure, en face des $\mathbf{1 . 0 0 0 ~ m o t s ~ d u ~ f a m e u x ~ a p p a r e i l ~}$ Hughes et des modestes 400 mots de l'appareil classique de Morse.

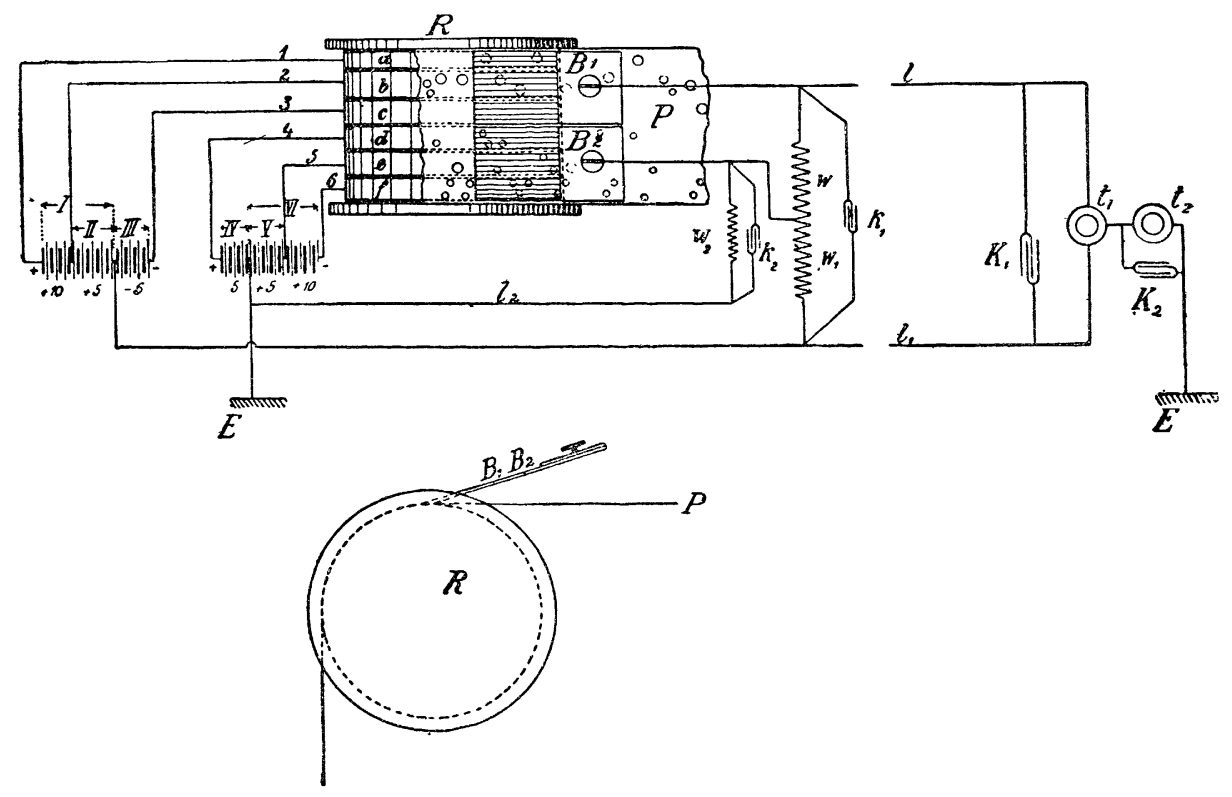

Fig. 1.

Pour obtenir de telles vitesses, il fallait un transmetteur à rotation rapide, où la main humaine devait se borner uniquement au rôle de préparation et de mise en marche, ainsi qu'un récepteur presque sans inertie pouvant suivre la rapidité de la transmission.

Pour le premier, les inventeurs ont eu recours à un moteur électrique qui met en rotation rapide un tambour métallique $\mathrm{R}(f g . \mathbf{1})$ sur lequel se déroule une bande de papier portant le texte du télégramme sous forme d'un système de trous perforés au moyen d'un instrument auxiliaire, le " perforateur ", complètement indépendant de l'appareil. 
L'aspect de ces perforations de grands et petits diamètres, qui se suivent sur la bande de papier, rappelle vaguement celui des signes de Morse. La bande de papier P est serrée par un rouleau contre un tambour métallique $\mathrm{R}(f g .1)$. Ce dernier est composé de six bagues juxtaposées $a, b, c, d, e, f$, isolées électriquement l'une de l'autre. Chaque bague est reliée métalliquement aux bornes correspondantes d'une batterie d'accumulateurs ou de piles sèches, déterminant ainsi pour chaque bague une autre différence de potentiel. Nous expliquerons plus loin la raison de la nécessité de ces divers degrés de potentiel auxdites bagues. Contre le ruban en papier sont serrés des

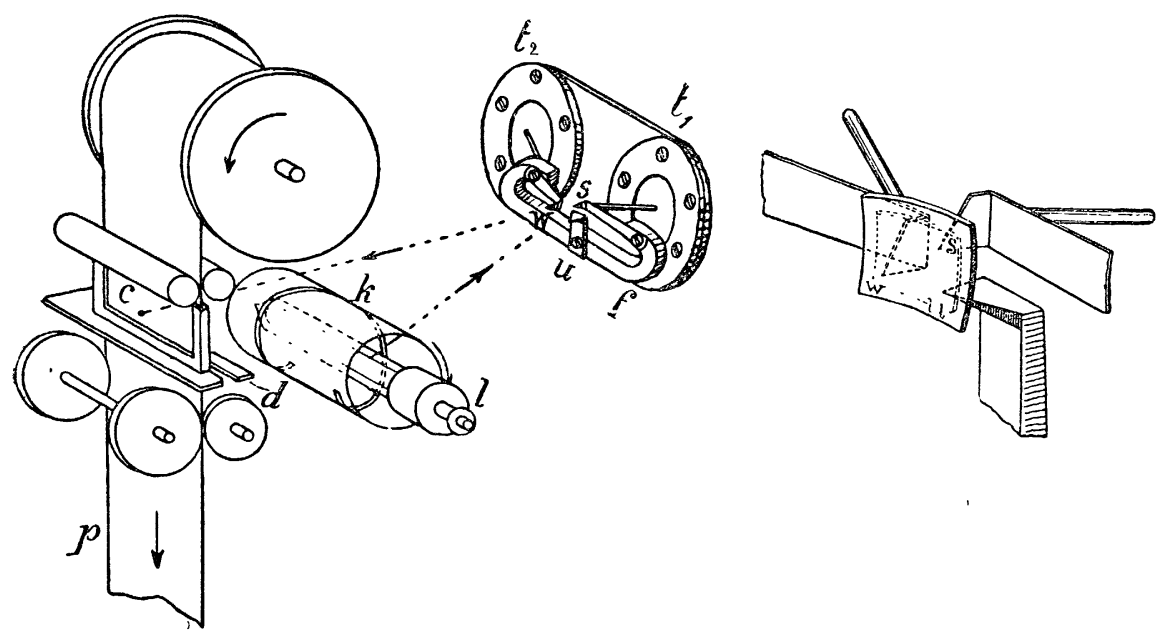

FIG. 2 et 3.

balais métalliques au nombre de deux fois trois, c'est-à-dire un par bague, et dont trois sont reliés en parallèle. $B_{1}$ représente la jonction des trois balais $a, b, c$, et $\mathrm{B}_{2}$ celle des trois autres $d, e, f$. Lorsque le balai, passant sur la bague correspondante du rouleau par-dessus le ruban de papier, arrive sur une perforation de celui-ci, la batterie envoie par ce balai, pendant le court instant du passage de ce dernier sur le trou, un courant dans la ligne. De cette façon, on peut transmettre environ 400 émissions de courant à la seconde, tandis que, par les procédés de télégraphie ordinaire, on n'arrive à en transmettre que $气$ ou 6 tout au plus.

Le récepteur, la partie la plus ingénieuse de l'invention, utilise ces 
émissions de courant, en les faisant agir sur les membranés de deux téléphones $t_{1}$ et $t_{2}(f g . \mathbf{1})$ et par leur intermédiaire sur un miroir ( $f g .2$ et 3 ) qui, en réfléchissant les rayons d'une lampe à incandescence, projette une tache lumineuse, un "spot", sur une bande de papier sensible. Comme le miroir repose sur un point fixe $u$, l'une des membranes téléphoniques $s$ lui imprime un mouvement vertical, tandis que l'autre $w$ le met en mouvement horizontal.

Par la combinaison de ces mouvements, le faisceau lumineux réfléchi écrit dans la chambre noire le texte du télégramme en caractères latins sur le papier photographique. Le développement de ce dernier se fait automatiquement dans un bain fixateur enfermé dans une partie spéciale de l'appareil, d'où le ruban insensibilisé, d'une largeur de 10 centimètres, sort, complètement achevé, prêt à être livré au destinataire. $d$ représente les ciseaux de l'appareil, servant à découper le papier aussitôt que le télégramme est inscrit.

Dans la première forme de leur appareil, les inventeurs n'ont eu recours qu'à un seul téléphone, et les télégrammes n'étaient encore reproduits que par une ligne ondulée, composée d'élongations hautes et moins hautes au moyen desquelles on pouvait combiner l'alphabet Morse, et rappelant les signes du siphon recorder de lord Kelvin (fig. 4).

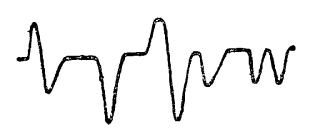

FIG. 4.

Nous pouvons remarquer qu'avec une légère transformation de cet appareil, rien qu'en remplaçant le transmetteur décrit plus haut par un microphone, nous pouvons obtenir sur le papier sensible une ligne ondulée représentant les variations du son produit devant le microphone. On a ainsi, comme MM. Pollak et Virag l'ont prouvé par les expériences exécutées à Budapest, une sorte de photographie de la voix.

C'est un tel appareil à un seul téléphone, donc incomplet, qui fut exposé à Paris en 1900 et qui fut expérimenté entre Berlin et Budapest, sur une ligne de 1.000 kilomètres de longueur, causant une grande sensation par la rapidité de la transmission. 
A la suite de ces expériences, les inventeurs ont été amenés à perfectionner leur invention en combinant le téléphone qui produit le mouvement vertical $d u$ " spot » avec un deuxième téléphone dit " à mouvement horizontal " et dont le fonctionnement simultané fournit comme résultat remarquable l'écriture en lettres ordinaires rappelant les figures lumineuses des célèbres expériences de M. Lissajous.

Le récepteur repose en somme sur le jeu d'un miroir qui envoie un rayon lumineux réfléchi sur le fond sensible d'une chambre noire et $y$ décrit des figures qui correspondent au mouvement imprimé. On pourrait s'imaginer une source lumineuse fixe et un miroir mobile ou bien - et c'est ce que les inventeurs ont préféré - une source de lumière mobile en même temps qu'un miroir qui vibre. En effet, dans leur appareil, le point lumineux est fourni par l'intersection du filament incandescent d'une lampe électrique et d'une fente hélicoïdale d'un diaphragme cylindrique $\mathrm{K}$ enveloppant cette lampe à incandescence $I$ et auquel le moteur de l'appareil imprime un mouvement de rotation synchrone. Il en résulte un mouvement rectiligne du point lumineux qui, après avoir décrit sa ligne droite, revient subitement à son point de départ et ainsi de suite. A ce mouvèment se superposent les vibrations du miroir, et il en résulte l'écriture lisible, dont chaque ligne est de la même largeur déterminée par la longueur de la fente du diaphragme et par la distance par rapport au papier sensible, et dont les lignes sont équidistantes en raison du mouvement d'avancement du papier par saccades qui se produisent au moment précis où le point lumineux est arrivé à la fin de chaque ligne.

Il y a encore une disposition ingénieuse à signaler pour le mode de suspension du miroir vibrant. En effet ce miroir est collé sur une petite plaque en fer (fig. 3) maintenue par l'attraction magnétique contre les trois pointes $u, w$ et $s$.

La pointe $u$ fait partie solidaire d'un aimant permanent en fer à cheval $f$ auquel les pointes $w$ et $s$ sont liées par de petits ressorts en fer (en fig. 2, le miroir a été enlevé pour faire voir la disposition des pointes). $w$ touche en même temps par une petite poignée la membrane du téléphone $t_{2}$, qui donne les traits horizontaux. et $s$ de même la membrane de $t_{1}$, donnant les traits verticaux.

Comme les traits horizontaux et verticaux doivent être de longueurs différentes pour les diverses parties composant les lettres de l'alphabet latin, les trois bagues $d, e, f$ du transmetteur actionnant 
le téléphone " horizontal " correspondent à des voltages différents. Ainsi $f$ correspond à +10 volts et fournit un trait long horizontal, $e$ correspond à $+\tilde{a}$ volts et donne un trait court horizontal, tandis que $d$ avec - $ّ$ volts fournit le même trait en sens inverse. De même, pour les traits verticaux, $a$ avec +10 volts donne un trait long produit par une grande élongation de la membrane du téléphone $t$; par contre, $b$ avec +5 volts ne donne qu'un trait moitié moins long, et $c$ avec - $ّ$ volts, ce même trait court en sens inverse. La fig. 1 fait voir également les schémas de fonctionnement électrique du transmetteur. Le téléphone " vertical " $t_{1}$ est insensible aux effets de courant venant des bagues $d$, $e, f$, qui correspondent au téléphone "horizontal " $t_{2}$. En effet le fil qui vient du balai $\mathrm{B}_{2}$ aboutit juste au milieu de la bobine $w$. Le courant se divise en deux fractions égales, qui viennent en sens inverse sur le téléphone $t_{1}$ et s'équilibrent comme effet sur la membrane de ce téléphone. Par contre, en passant par le téléphone $t_{2}$ à la terre $\mathrm{E}$, elles font fonctionner la membrane de cet appareil $t_{2}$.

ll y a diverses circonstances dont il faut tenir compte soigneusement, si l'on veut éviter des déformations gênantes dans les caractères de l'écriture. Ainsi, par exemple, il est de toute évidence que les membranes des téléphones, en même temps qu'elles suivront les impulsions de courant, seront soumises également aux effets de'leurs vibrations propres susceptibles de nuire beaucoup à la netteté des lettres.

On pourrait éliminer ces perturbations en donnant aux impulsions de courant la même durée que celle de la période de vibration de la membrane téléphonique, c'est-à-dire en choisissant la vitesse du ruban de papier et le diamètre des trous de contact de telle façon que la durée du contact métallique entre les balais et les disques coïncide avec ladite période. Dans ce cas, la force motrice qui agit sur la membrane cesserait au moment où cette dernière passe par sa position d'équilibre.

Les oscillations nuisibles deviennent alors très faibles et s'amortissent rapidement.

Les inventeurs ont même préféré choisir la durée des contacts plus petite que la période d'oscillation propre des membranes et intercaler plutôt des condensateurs $K_{1}$ et $K_{2}$ dans les circuits en parallèle avec les téléphones. En effet ces condensateurs peuvent se charger pendant la durée du contact et se décharger ensuite dans la 
bobine du téléphone, aussitôt que le mouvement continu du ruban de papier aura interrompu le contact métallique. Ce courant de décharge achève donc l'élongation de la membrane; mais en mêmé temps, si la capacité du condensateur est bien choisie, elle assure les conditions nécessaires pour que le mouvement amorti de la membrane soit apériodique et non pas oscillatoire.

Une autre circonstance dont il faut tenir compte résulte de la haute capacité et de la forte résistance des longues lignes télégraphiques qui, pour des courants de hautes fréquences aussi rapides que ceux produits par l'appareil Pollak et Virag, donneraient lieu à une constante de temps trop élevée, c'est-à-dire à une propagation trop lente du courant et, par conséquent, à une déformation de l'écriture. Pour y remédier, on a intercalé les bobines de self-induction $w_{1}, v_{2}, w_{3}$. Mais non seulement l'établissement du courant devient plus rapide par la présence de ces bobines, mais en mème temps l'extinction également. En effet, sans ces bobines, la capacité des lignes occasionnerait une décharge lente, tandis que le courant de self-induction produit par ces bobines au moment de la rupture de contact, et qui se décharge dans le circuit du téléphone en direction opposée à celle du courant principal, éteint rapidement ce dernier et rend la membrane apte à recevoir immédiatement une nouvelle impulsion de la part du contact suivant, qui est dû à la perforation suivante du ruban de papier.

Comme les traits horizontaux exigent des impulsions plus lentes que les traits verticaux, on peut se contenter pour le téléphone " horizontal » d'une bobine de self $w_{2}$ plus petite que pour la membrane verticale.

Les inventeurs ont fait fonctionner leur appareil avec ou sans ces bobines de self et ont prouvé ainsi la grande influence que la présence de ces bobines exerce sur la netteté des caractères.

Cette dernière est enfin affectée par la différence de phase existant entre les impulsions " horizontale " et " verticale ». En effet la durée du traçage entre un trait horizontal et le trait vertical qui suit ne peut pas être quelconque, mais bien correspondante aux caractères qui sont à tracer. On a essayé d'abord de régler cette durée par le réglage des balais; toutefois il a paru plus efficace de recourir à l'emploi du condensateur $k_{1}$ et $k_{2}$, dont les capacités sont choisies de façon à produire juste la différence de phase nécessaire.

Par ces moyens les effets perturbateurs sont complètement anni- 
hilés. Les autres perturbations qui peuvent résulter de l'effet d'induction des fils de ligne voisins ou des courants terrestres peuvent être évitées au moyen de téléphones construits spécialement à cet effet en vue d'y être insensibles.

En ce qui concerne les détails techniques du télégraphe PollakVirag, il nous reste à dire un mot de l'appareil qui sert à préparer le ruban perforé.

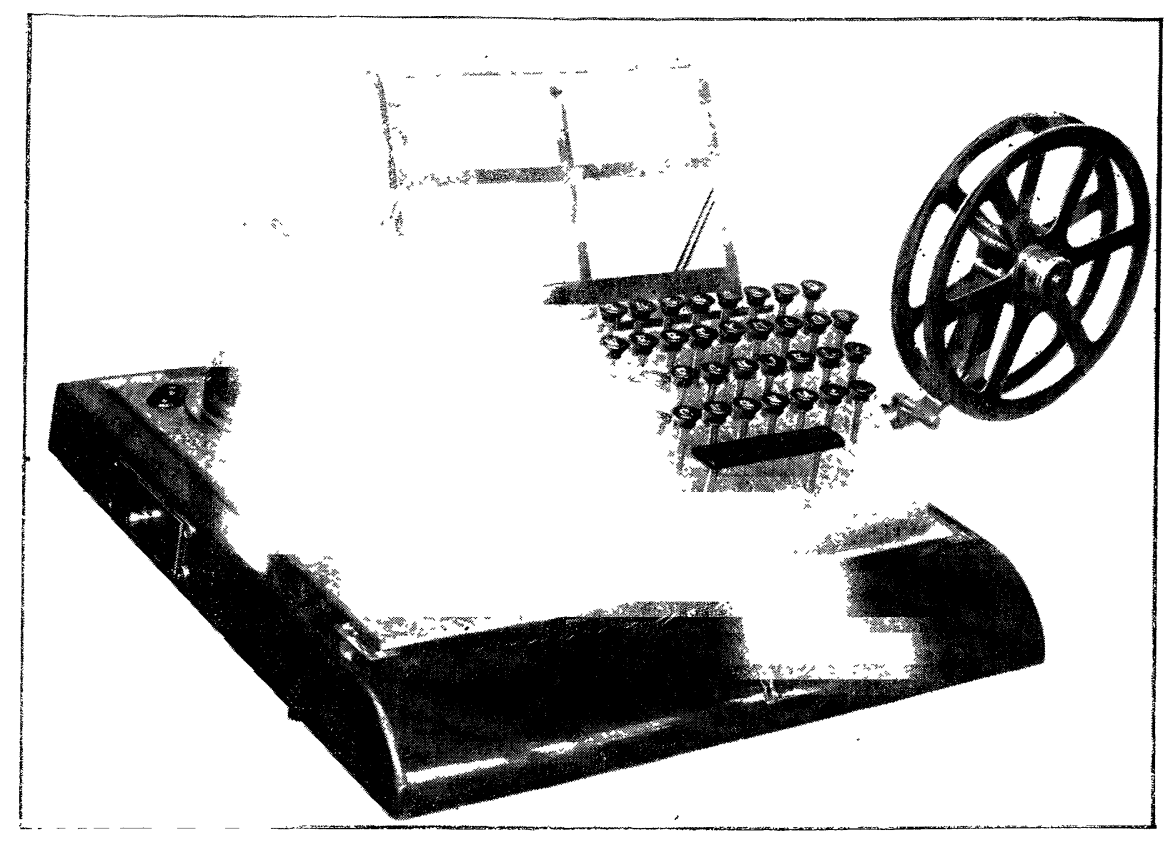

FIG.

Cet appareil perforateur est représenté sur la fig. ̋̃. Il consiste en une machine à écrire dont les touches font avancer par un mouvement horizontal un certain nombre de barrettes sur lesquelles des aiguilles perforatrices peuvent alors s'appuyer. Le ruban $a$, mû par l'organe entraineur $b$, en passant sous l'outil $c$ qui est abaissé par l'électro-aimant $e$, reçoit donc autant de trous à chaque mouvement de touches que le nombre de barrettes avancées et bloquées par la. touche l'indique. A chaque lettre à imprimer correspond ainsi une autre disposition des trous.

J. de Phys., 4 série, t. VI. (Avril 1907.) 
Il est évident que les rubans perforés peuvent être préparés indépendamment de l'appareil télégraphique. L'expéditeur, qui possède un appareil perforateur chez lui, n'a donc qu'à apporter le ruban perforé à l'employé du télégraphe. Pour nous faire une idée de la capacité du système, nous pouvons rappeler que vingt-trois machines à écrire perforatrices suffisent à peine pour alimenter un appareil télégraphique Pollak-Virag en plein fonctionnement continu.

En effet on est arrivé à perforer 2.000 mots par heure avec un appareil perforateur, tandis qu'un appareil télégraphique PollakVirag peut transmettre près de 50.000 mots.

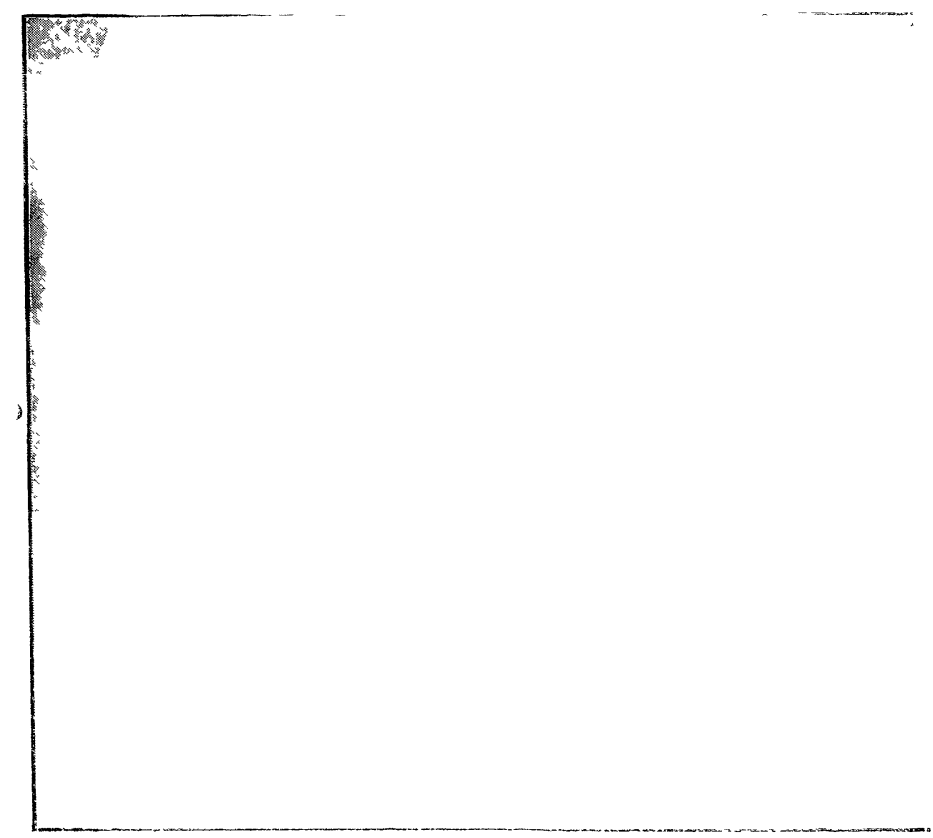

Fig. 6.

Voici maintenant comment fonctionne le système dans la pratique: Dès que l'employé préposé au poste $\mathrm{A}$ veut correspondre avec le poste B, il appelle ce dernier en agissant par la poignée $b$ ( $f g .6$ ) du transmetteur sur un inducteur par lequel la station réceptrice est mise en circuit. Au préalable, il aura ajusté son ruban perforé sur l'organe d'entraînement $a$ que l'interripteur $c$ du moteur électrique 
permet de mettre en mouvement; $d, d$ sont des résistances, et $e, e$ les condensateurs dı transmelteur. L'employé de la station $\mathrm{B}$, une fois prévenı, met en fonctionnement son moteur élec-
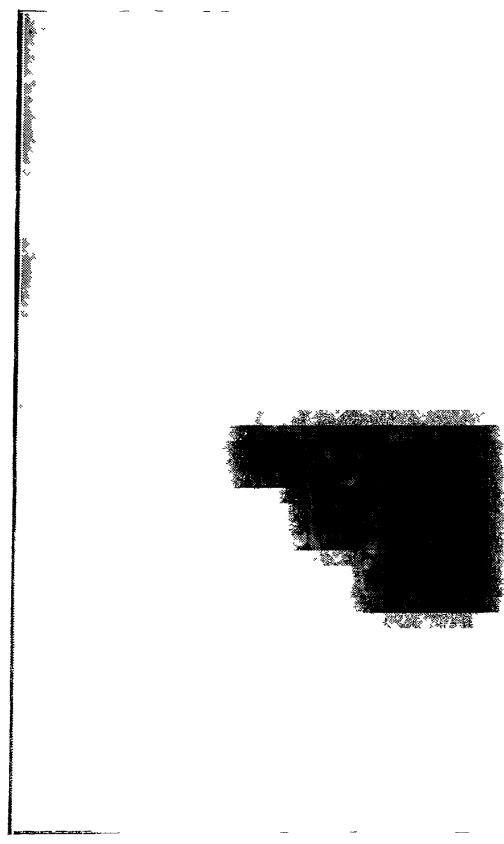

Fig. T.

trique, en agissant sur le 'régulateur $C(f g .7)$ du mécanisme de commande du récepleur. Le reste se fait automatiquement. En effet l'inducteur du poste A, en envoyant le signal d'avertissement, a saisi au moyen d'un électro-aimant, qui ağit sur l'organe $a$, le papier sensible dont la quantite est indiquée par l'aiguille $b$, et le met en mouvement; $c$ est une lenêtre garnie d'un verre rouge permettant de voir les déplacements du point lumineux que l'on peut suivre comme une sorte de flamme traçant des leltres.

Le message étant terminé, la bande est coupée parle couteau $d$ et n'a plus alors qu'à passer dans les bains photographiques. $t$ indique la paire de téléphones en face desquels se meut le miroir qui réfléchit le rayon qui écrit.

Une lentille $g$ ajustable par une vis $h$ est placée devant le miroir 
pour des motifs d'ordre optique. $i$ est un interrupteur; $l$, un commutateur pour un fil de conversation, et $m$, un tendeur pour le fil de transmission.

La $f g .8$ représente un spécimen d'une dépêche ainsi transmise, dont le ruban perforé est reproduit en $f g .9$.

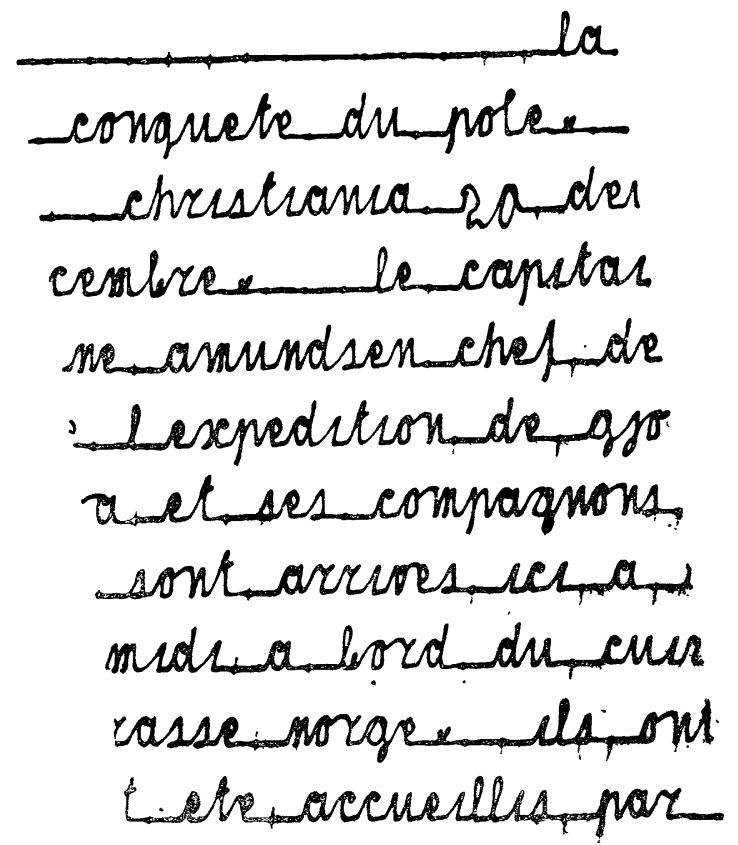

FIG. 8.

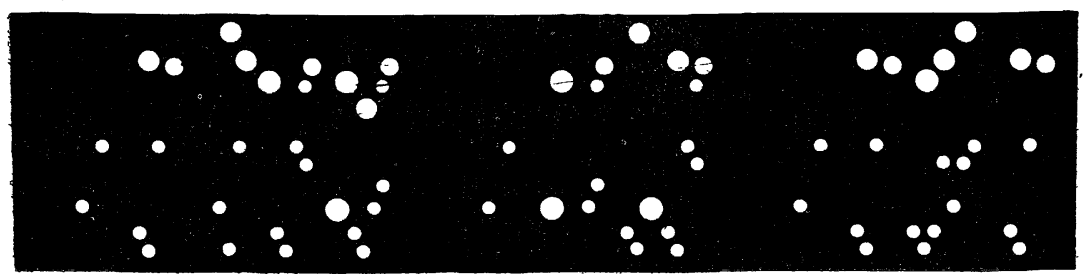

FIG. 9.

Ce système, qui peut répondre à toutes les exigences, a fait ses preuves dans la pratique à divers essais prolongés officiels; ainsi qu'en témoignent les attestations flatteuses du Gouvernement hon- 
grois pour les essais faits entre Budapest et Pozsony (Presbourg), sur une ligne de 218 kilomètres, ainsi que celle du Gouvernement allemand pour les essais entre Berlin et Kœnigsberg, sur une ligne de 710 kilomètres.

Le document hongrois est surtout élogieux et indique aussi pour quel motif cette ingénieuse invention hongroise n'a pas encore triomphé dans sa patrie.

En effet il n'existe pas actuellement deux bureaux dans le pays ayant entre eux un trafic suffisant pour fournir une quantité de travail convenable justifiant l'installation de l'appareil Pollak-Virag.

Quelques altérations dans les lignes téléphoniques, provenant de la mauvaise disposition de ces lignes, furent remarquées dans les premières expériences allemandes, mais dans les expériences entre Berlin et Francfort-sur-Mein, sur une longueur de 550 kilomètres, rien de pareil ne fut constaté; de même les expériences qui ont eu lieu l'année dernière pendant plusieurs mois entre Paris et Lyon n'ont donné lieu à aucun trouble sérieux.

Nous pouvons ajouter que les expériences ont eu lieu sur deux lignes Paris-Lyon : l'une de 522 kilomètres, en fil de 4 millimètres de diamètre, résistance $700 \mathrm{ohms,} \mathrm{capacité} 7 /$ o microfarad; l'autre de 519 kilomètres, en fil de $3 / \check{3}$ millimètres, résistance $487 \mathrm{ohms}$, capacité 4/664 microfarad. Les deux fils étaient donc suffisamment différents, et néanmoins aucun trouble ne fut constaté.

Si nous passons maintenant à l'examen des conditions de service pratique du système Pollak-Virag, il nous faut tout d'abord remarquer que l'on ne peut pas employer avec ce système des lignes de fil de fer en usage avec les appareils télégraphiques actuels.

En effet de telles lignes, comme on peut bien le penser, ne permettent pas le passage de 300 à 400 émissions de courant par seconde, c'est-à-dire un nombre analogue à celui d'une communication téléphonique.

Il faut donc recourir à des lignes téléphoniques en cuivre ou bronze siliceux pour la transmission des télégrammes rapides. Nous nous empressons d'ajouter qu'il n'est pas néçessaire que ces lignes soient préservées du phénomène d'induction comme devraient l'être les lignes téléphoniques, car les appareils Pollak-Virag, ainsi que l'on peut s'en rendre compte par les schémas représentés plus haut, possèdent des téléphones dans lesquels les effets d'induction s'éliminent par la disposition même des circuits. Ils sont donc rela- 
tivement insensibles aux influences inductives, comme d'ailleurs les expériences précitées, surtout celles faites dans ce but en Allemagne entre Berlin et Francfort-sur-Mein, sur une longueur de כّ̋0 kilomètres, l'ont prouvé.

Toutefois, les avantages économiques du syslème ne sont pas altérés par l'emploi de lignes de cuivre, car, en réalité, le système Pollak-Virag procure une considérable économic sur l'établissement des lignes, puisquil permet, avec deux fils, de transmettre une même quantité de mots que le système Hughes, par exemple, permet à peine en employant 20 fils.

L'économie principale du système Pollak-Virag réside cependant surtout dans la réduction considérable de personnel et de maind'œuvre. Pour nous en rendre compte, examinons le tableau suivant, qui offre une comparaison des quatre systèmes actuellement en usage: Morse, Hughes, Baudot et Pollak-Virag, en ce qui concerne leur production, leur capacité et le prix de revient de la transmission des dépêches, à l'aide de chacun de ces systèmes.

Il a été admis, comme base du calcul, que 6.000 mots taxés doivent être transmis entre deux stations par heure; cela fait, avec les préambules, appels, ordres de service, etc., en réalité 11 à 12.000 mots à transmettre effectivement. Les dépenses, pour l'entretien de la ligne de õ00 kilomètres de longueur et l'intérèt du capital investi, sont évaluées à 6.000 francs et les frais par employé à 2.400 francs par an, et par auxiliaire à 1.5000 francs par an.

Pour le système Morse, ainsi que pour l'appareil Hughes, il faut deux employés par ligne, tandis que pour le Baudot, où 4 appareils peuvent travailler en même temps sur une ligne pour transmettre 5 à 6.000 mots à l'heure, il faut huit employés et deux dirigeurs, soit, pour les deux lignes nécessaires avec deux quadruples, vingt personnes, sans compter les six auxiliaires chargés des expéditions, enregistrement, etc. Pour le système Pollak-Virag, il faut le travail simultané de l'employé transmetteur, de l'employé récepteur et le sept employés perforateurs, ainsi que de six auxiliaires. Commel'appareil a une capacité de 40.000 mots à l'heure, il en résulte que, pour 6.000 mots taxés, soit 12.000 mots effectils à l'heure, les employes transmetteur et récepteur resteront libres pendant $28 / 40$, soit environ trois quarts d'heure, pour aider à perforer les rubans des dépêches différentes. Il est également à remarquer que, dans le cas où une partie de la grosse clientèle, comme, par exemple, la presse et la 
banque, préparerait chez elle les rubans perforés, en les apportant à la place des textes écrits au bureau du télégraphe, le nombre des employés perforateurs pourrait être encore réduit dans une forte proportion.

Mais, même avec les conditions envisagées, le tableau démontre la grande supériorité suivante du système Pollak-Virag :

\begin{tabular}{|c|c|c|c|c|c|c|c|}
\hline \multirow{3}{*}{ Système } & \multirow{3}{*}{$\begin{array}{c}\text { Production } \\
\text { et } \\
\text { capacité } \\
\text { en mots } \\
\text { par apparell } \\
\text { et par heure }\end{array}$} & \multicolumn{6}{|c|}{ La transmission de 6.000 mots taxés à l'heüre exige } \\
\hline & & \multicolumn{5}{|c|}{ Nombres de dépenses pour } & \multirow{2}{*}{$\begin{array}{l}\text { Total } \\
\text { par an } \\
\text { francs }\end{array}$} \\
\hline & & lignes & employés & $\begin{array}{c}\text { auxi- } \\
\text { liaires }\end{array}$ & lignes & $\begin{array}{l}\text { employés } \\
\text { et auxiliaires }\end{array}$ & \\
\hline Morse.. & 500 à 2.000 & 20 & 40 & 6 & 120.000 & $10: 3.000$ & 225.000 \\
\hline Hughes.. & 1.000 à 1.200 & 10 & 20 & 6 & 60.000 & 37.000 & 117.000 \\
\hline Baudot...... & 5.000 à 6.000 & 2 & 20 & 6 & 12.000 & 57.000 & 59.000 \\
\hline Pollak-Virag. & 40.000 & 2 & 2 & 13 & 12.000 & 24.300 & 35.300 \\
\hline
\end{tabular}

Ce tableau serait encore plus favorable si I'on pouvait l'appliquer à une ligne d'un trafic beaucoup plus intense, parce que le système Pollak-Virag permet l'emploi d'une main-d'œuvre à bon marché.

Il en résulte en définitive la possibilité d'une réduction très notable des tarifs. Or cette dernière amènera la création de nouvelles sources de trafic et de recettes qu'il nous est impossible de passer sous silence. Nous voulons parler des lettres-télégrammes qui sont presque impossibles sous l'ancien régime, ainsi que des dépêches circulaires, la même dépêche pouvant être expédiée simultanément dans n'importe combien de directions et de stations distinctes en quelques secondes au moyen du même ruban perforé, sans autre travail manuel ni préparation ultérieure.

En considérant tous ces faits, nous devons reconnaître que le télégraphe rapide Pollak-Virag constitue une invention fort ingénieuse, qui est pleine d'avenir et peut avoir de grandes conséquences à tous égards. 